\title{
Clean coalgebras and clean comodules of finitely generated projective modules*
}

\author{
N. P. Puspita, I. E. Wijayanti, and B. Surodjo
}

Communicated by R. Wisbauer

\begin{abstract}
Let $R$ be a commutative ring with multiplicative identity and $P$ is a finitely generated projective $R$-module. If $P^{*}$ is the set of $R$-module homomorphism from $P$ to $R$, then the tensor product $P^{*} \otimes_{R} P$ can be considered as an $R$-coalgebra. Furthermore, $P$ and $P^{*}$ is a comodule over coalgebra $P^{*} \otimes_{R} P$. Using the Morita context, this paper give sufficient conditions of clean coalgebra $P^{*} \otimes_{R} P$ and clean $P^{*} \otimes_{R} P$-comodule $P$ and $P^{*}$. These sufficient conditions are determined by the conditions of module $P$ and $\operatorname{ring} R$.
\end{abstract}

\section{Introduction}

In this paper a commutative ring with the identity is denoted by $R$. A ring $R$ is said to be a clean ring if every element of $R$ can be express as a sum of a unit and an idempotent element [1]. Moreover, a clean ring is one of the subclasses of exchange rings $[2,3]$. The previous authors have given some notions of clean rings and exchange rings for example [4-9].

Some authors have been studied the endomorphism structure of $R$ modules $M$. It is proved that the ring of a linear transformation of a countable linear vector space is clean [10] and the result is also true for arbitrary vector spaces over a field and any vector space over a division

${ }^{*}$ This research is supported by Doctoral Research Grant, Directory of Higher Education, the Ministry of Education and Culture, Indonesia, 2019-2020.

2020 MSC: 16T15, 16D90, 16D40.

Key words and phrases: clean coalgebra, clean comodule, finitely generated projective module, Morita context. 
ring, it is has been proved in [11] and [12]. An $R$-module $M$ is called a clean

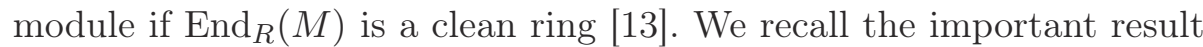
of [13], i.e., necessary and sufficient conditions of clean elements in an endomorphism ring (see Proposition 2.2 and Proposition 2.3). Furthermore in [14] the authors prove this property in a shorter way by proving that every non $M$-singular self-injective module $M$ is clean (see Lemma 4).

The structure of comodules and coalgebras has been introduced in 1969 by Sweedler. He introduced a coalgebra over a field as the dualization of algebras over a field. Later, this ground field has been generalized to any ring with multiplicative identity [15]. Furthermore, a comodule over a coalgebra is well-known as a dualization of a module over a ring. For any $R$-coalgebra $C$ we can construct $C^{*}=\operatorname{Hom}_{R}(C, R)$, where $C^{*}$ is an algebra (ring) over convolution product. We called $C^{*}$ as a dual algebra of $C$. Hence, we have an important result, i.e. if $M$ is a right $C$-comodule, then $M$ is a left module over the dual algebra $C^{*}$. Moreover, for any $M, N \in \mathbf{M}^{\mathbf{C}}$ and $\operatorname{End}^{C}(M, N) \subseteq C^{*} \operatorname{End}(M)$. Thus, the category of right $C$-comodule $\left(\mathbf{M}^{\mathbf{C}}\right)$ is a subcategory of left $C^{*}$-module $\left(C^{*} \mathbf{M}^{\mathbf{C}}\right)$. In [15], the $R$-coalgebra $C$ satisfies the $\alpha$-condition if and only if $\mathbf{M}^{\mathbf{C}}$ is a full subcategory of $C^{*} \mathbf{M}^{\mathbf{C}}$. Moreover, the $\operatorname{End}^{C}(M)=C^{*} \operatorname{End}(M)$ if and only if $C$ is locally projective as an $R$-module (see [15]).

Recall the structure of comodules and coalgebras [15]. We applied the notions of clean modules to comodule and coalgebra, and introduced the following definition.

Definition 1. Let $R$ be a ring and $(C, \Delta, \varepsilon)$ an $R$-coalgebra. A right (left) $C$-comodule $M$ is called a clean comodule if the endomorphism ring of right (or left) $C$-comodule $M$ (denoted by $\operatorname{End}^{C}(M)\left(\right.$ or $\left.{ }^{C} \operatorname{End}(M)\right)$ is a clean ring.

Definition 1 means that if $C$ satisfies the $\alpha$-condition, the right $C$ comodule $M$ is a clean comodule if and only if the $\operatorname{ring} C^{*} \operatorname{End}(M)$ is a clean ring, since $\operatorname{End}^{C}(M)=C^{*} \operatorname{End}(M)$. Since every $R$-coalgebra $C$ is a right and left comodule over itself, based on Definition 1 we introduce a clean coalgebra.

Definition 2. Let $R$ be a ring. An $R$-colagebra $C$ is called a clean coalgebra if $C$ is a clean comodule over itself.

If $C$ satisfies the $\alpha$-condition, Definition 2 means that $C$ is a clean coalgebra if $C$ is clean as a $C^{*}$-module. We present the trivial of clean coalgebra. Consider any ring $R$ as an $R$-coalgebra with the trivial comultiplication $\Delta_{T}: R \rightarrow R \otimes_{R} R, r \mapsto r \otimes r$, and counit $\varepsilon_{T}: R \rightarrow R$, 
$r \mapsto r$, for any $r \in R$. Hence, the dual algebra of $R$, i.e., $\left(R^{*},+, *\right)$ where $R^{*}=\operatorname{End}_{R}(R)$ is isomorphic to the ring $R$ by mapping $f \mapsto f(1)$ for all $f \in R^{*}$. Then we have a trivial $R$-coalgebra $\left(R, \Delta_{T}, \varepsilon_{T}\right)$ is a clean if and only if $R$ is a clean ring.

Furthermore, since every ring $R$ can be considered as the trivial $R$ coalgebra $\left(R, \Delta_{T}, \varepsilon_{T}\right)$, any $R$-module $M$ is a (right and left)comodule over coalgebra $\left(R, \Delta_{T}, \varepsilon_{T}\right)$ with coaction

$$
\varrho^{M}: M \mapsto M \otimes_{R} R, m \mapsto m \otimes 1 .
$$

It implies any $R$-module $M$ is clean if and only if $\left(M, \varrho^{M}\right)$ is a clean (right and left) $R$-comodule, since $R \simeq R^{*}$.

Throughout $P$ is a finitely generated (f.g) projective $R$-module and $P^{*}$ is a set of all $R$-module homomorphism from $P$ to $R$. In [15] we have already know that for any f.g projective module $P$ and $P^{*}$, we can construct tensor product of $P$ and $P^{*}$, i.e., $P^{*} \otimes_{R} P$. An $R$-module $P^{*} \otimes_{R} P$ is an $R$-coalgebra by a comultiplication $\Delta$ and counit $\varepsilon$ as below:

Lemma 1. [15] Let $P$ be a finitely generated projective $R$-module with dual basis $p_{1}, p_{2}, \ldots, p_{n} \in P$ and $\pi_{1}, \pi_{2}, \ldots, \pi_{n} \in P^{*}$. The $R$-module $P^{*} \otimes_{R} P$ is an $R$-coalgebra with the comultiplication and counit defined by

$$
\begin{aligned}
\Delta: P^{*} \otimes_{R} P & \rightarrow\left(P^{*} \otimes_{R} P\right) \otimes_{R}\left(P^{*} \otimes_{R} P\right) ; \\
f \otimes p & \mapsto \Sigma_{i} f \otimes p_{i} \otimes \pi_{i} \otimes p
\end{aligned}
$$

and

$$
\varepsilon: P^{*} \otimes_{R} P \rightarrow R, \quad f \otimes p \mapsto f(p) .
$$

By the properties of the dual basis,

$$
\left(I_{P^{*} \otimes_{R} P} \otimes \Delta\right) \varepsilon(f \otimes p)=\Sigma_{i} f \otimes p_{i} \pi_{i}(p)=f \otimes p,
$$

that is $\varepsilon$ is a counit and the coassociativity of $\Delta$ is proved by the following equality

$$
\begin{aligned}
\left(I_{P^{*} \otimes_{R} P} \otimes \Delta\right) \Delta(f \otimes p) & =\Sigma_{i, j} f \otimes p_{i} \otimes \pi_{i} \otimes p_{j} \otimes \pi_{j} \otimes p \\
& =\left(\Delta \otimes I_{P^{*} \otimes_{R} P}\right) \Delta(f \otimes p) .
\end{aligned}
$$

Furthermore, consider $P$ and $P^{*}$ as an $R$-module, then $P$ and $P^{*}$ respectively can be consider as a right and left comodule over $R$-coalgebra $P^{*} \otimes_{R} P$. By using the Morita context, which is we refer to [16], in this paper we investigate the sufficient conditions of clean $R$-coalgebra $P^{*} \otimes_{R} P$ 
and the cleanness of $P$ and $P^{*}$ as a $P^{*} \otimes_{R} P$-comodule. In Morita context we already know there are relationship between the structure of $P, P^{*}, R$ and $S=\operatorname{End}_{R}(P)$ [16]. The following theorem explain the relationship between $P$ and its dual, in which it is important to prove our main result.

Theorem 1. [16] Let $R$ be a ring, $P$ be a right $R$-module, $S=\operatorname{End}_{R}(P)$ and $Q=P^{*}=\operatorname{Hom}_{R}(P, R)$. If $P$ is a generator in $R$-MOD, then

1) $\alpha: Q \otimes_{S} P \rightarrow R$ is an $(R, R)$-isomorphism;

2) $Q \simeq \operatorname{Hom}_{S}\left({ }_{S} P,{ }_{S} S\right)$ as $(R, S)$-bimodules;

3) $P \simeq \operatorname{Hom}_{S}\left(Q_{S}, S_{S}\right)$ as $(S, R)$-bimodules;

4) $R \simeq \operatorname{End}\left({ }_{S} P\right) \simeq \operatorname{End}\left(Q_{S}\right)$ as rings.

Theorem 2. [16] Let $R$ be a ring, $P$ be a right $R$-module, $S=\operatorname{End}_{R}(P)$ and $Q=P^{*}=\operatorname{Hom}_{R}(P, R)$. If $P$ is finitely generated projective in $R$-MOD, then

1) $\beta: P \otimes_{R} Q \rightarrow S$ is an $(S, S)$-isomorphism;

2) $Q \simeq \operatorname{Hom}_{R}\left(P_{R}, R_{R}\right)$ as $(R, S)$-bimodules;

3) $P \simeq \operatorname{Hom}_{R}\left({ }_{R} Q,{ }_{R} R\right)$ as $(S, R)$-bimodules;

4) $S \simeq \operatorname{End}\left(P_{R}\right) \simeq \operatorname{End}\left({ }_{R} Q\right)$ as rings.

Since the cleanness of coalgebra and comodule are determined by the structure of its endomorphism, using Theorem 2 and Theorem 1, we observe when $\operatorname{End}_{\left(P^{*} \otimes_{R} P\right)^{*}}\left(P^{*} \otimes_{R} P\right), \operatorname{End}_{\left(P^{*} \otimes_{R} P\right)^{*}}(P)$ and $\operatorname{End}_{\left(P^{*} \otimes_{R} P\right)^{*}}\left(P^{*}\right)$ are clean.

\section{The clean $R$-coalgebra $P^{*} \otimes_{R} P$}

Let $P$ be an $R$-module. Here, we can construct tensor product of $P$ and $P^{*}$. Furthermore, since $R$ is a commutative ring, $P^{*} \otimes_{R} P \cong P \otimes_{R} P^{*}$ as an $R$-module. In this section we give some results which are related to some conditions when the $R$-coalgebra $P^{*} \otimes_{R} P$ is clean. Let $P$ be a finitely generated projective $R$-module with basis $p_{1}, p_{2}, \ldots, p_{n} \in P$ and dual basis $\pi_{1}, \pi_{2}, \ldots, \pi_{n} \in P^{*}$. Based on Theorem 2 we have $P \otimes_{R} P^{*} \cong \operatorname{End}_{R}(P)$ as an $(S, S)$-bimodule where

$$
P \otimes_{R} P^{*} \rightarrow \operatorname{End}_{R}(P), p \otimes f \mapsto[a \mapsto p f(a)] .
$$

Now, consider the $R$-module $P^{*} \otimes_{R} P$ as an $R$-coalgebra, using the Morita Context we have the following proposition.

Theorem 3. Let $P$ be a finitely generated projective $R$-module with dual basis $p_{1}, p_{2}, \ldots, p_{n} \in P \pi_{1}, \pi_{2}, \ldots, \pi_{n} \in P^{*}$. If $P$ is a clean $R$-module, then the $R$-coalgebra $P^{*} \otimes_{R} P$ is clean. 
Proof. Let $P$ be a finitely generated $R$-module and $P^{*}=\operatorname{Hom}_{R}(P, R)$ is an $R$-module. Suppose that $P$ is a clean $R$-module. Since $P$ and $R$ is a finitely generated projective $R$-module, $P^{*}=\operatorname{Hom}_{R}(P, R)$ is also a finitely generated projective $R$-module [17]. Here, we need to prove weather $R$-coalgebra $P^{*} \otimes_{R} P$ satisfies the $\alpha$-condition by proving the tensor product of $P^{*} \otimes_{R} P$ is a projective $R$-module.

To show that $P^{*} \otimes_{R} P$ is projective as an $R$-module, we must show that for any surjective map $f: A \rightarrow B$ of $R$-module, the map

$$
f_{*}: \operatorname{Hom}_{R}\left(P^{*} \otimes_{R} P, A\right) \rightarrow \operatorname{Hom}_{R}\left(P^{*} \otimes_{R} P, B\right)
$$

is also surjective. Since $P^{*}$ is a projective $R$-module so that

$$
h: \operatorname{Hom}_{R}\left(P^{*}, A\right) \rightarrow \operatorname{Hom}_{R}\left(P^{*}, B\right)
$$

is surjective. By projectivity of $P$ we obtain

$$
h_{*}: \operatorname{Hom}_{R}\left(P, \operatorname{Hom}_{R}\left(P^{*}, A\right)\right) \rightarrow \operatorname{Hom}_{R}\left(P, \operatorname{Hom}_{R}\left(P^{*}, B\right)\right)
$$

is also surjective. Put $C=A$ or $B$, then by [17] (see page 425) we have

$$
\operatorname{Hom}_{R}\left(P, \operatorname{Hom}_{R}\left(P^{*}, C\right)\right) \simeq \operatorname{Hom}_{R}\left(P^{*} \otimes_{R} P, C\right)
$$

It implies that $f$ is isomorphic to $h_{*}$, and moreover $f$ is a surjective map. Thus, $P^{*} \otimes_{R} P$ is a projective $R$-module. Therefore as an $R$-coalgebra, $P^{*} \otimes_{R} P$ satisfies the $\alpha$-condition. Then we have

$$
\left(P^{*} \otimes_{R} P\right)^{*} \operatorname{End}\left(P^{*} \otimes_{R} P\right) \simeq\left(P^{*} \otimes_{R} P\right)^{*} .
$$

We are going to show that $P^{*} \otimes_{R} P$ is a clean $R$-coalgebra, it means we need to prove that $\left(P^{*} \otimes_{R} P\right)^{*}$ is a clean ring (see Proposition 4.1.8). Based on [17], we have a relationship between tensor product and $R$ module homomorphism. Furthermore, since $P$ is finitely generated, the dual algebra $P^{*} \otimes_{R} P$ is isomorphic to the ring $\operatorname{End}_{R}(P)$ by the bijective map as below:

$$
\begin{aligned}
\left(P^{*} \otimes_{R} P\right)^{*} & =\operatorname{Hom}_{R}\left(P^{*} \otimes_{R} P, R\right) \simeq \operatorname{Hom}_{R}\left(P, \operatorname{Hom}_{R}\left(P^{*}, R\right)\right) \\
& \simeq \operatorname{Hom}_{R}\left(P, P^{* *}\right) \simeq \operatorname{End}_{R}(P)\left(\text { since } P^{* *} \simeq P\right) .
\end{aligned}
$$

Hence, if $P$ is a clean $R$-module, then $\operatorname{End}_{R} P$ is a clean ring. It means $\left(P^{*} \otimes_{R} P\right)^{*} \simeq \operatorname{End}_{R}(P)$ is a clean ring. Since $P^{*} \otimes_{R} P^{*} \simeq$ $\operatorname{End}_{\left(P^{*} \otimes_{R} P\right)^{*}}\left(P^{*} \otimes_{R} P\right)$ is a clean ring, $P^{*} \otimes_{R} P$ is a clean $R$-coalgebra. 
For $P=R$ we obtain $P^{*}=R^{*}=\operatorname{End}_{R}(R) \simeq R$ and $R^{*} \otimes_{R} R \simeq R$ is a coassociative $R$-coalgebra with counital. Thus, if $R$ is a clean $R$-module (i.e., $R$ is clean as a ring), then $(R, \Delta, \varepsilon)$ is a clean coalgebra over itself.

Recall the example of $R$-coalgebra $M_{n}(R)$ (see [15]). The matrix ring $M_{n}(R)$ is an $R$-coalgebra by the coproduct and counit as below

$$
\Delta: M_{n}(R) \rightarrow M_{n}(R) \otimes_{R} M_{n}(R), e_{i j} \mapsto \Sigma_{i, j} e_{i, k} \otimes e_{k j},
$$

and

$$
\varepsilon: M_{n}(R) \rightarrow R, e_{i j} \mapsto \delta_{i, j}
$$

It is called the $(n, n)$-matrix coalgebra over $R$. Throughout, the $R$ coalgebra $M_{n}(R)$ with the comultiplication (1) and the counit (2) denoted by $M_{n}^{C}(R)$. Furthermore, we will show that the $R$-coalgebra $M_{n}^{C}(R)$ can be identified as an $R$-coalgebra $P^{*} \otimes_{R} P$ when $P=R^{n}$.

Lemma 2. Let $P=R^{n}$. Then the comultiplication and counit on $R$ coalgebra $\left(R^{n}\right)^{*} \otimes_{R} R^{n}$ is equivalent to the comultiplication and counit of $R$-coalgebra $M_{n}^{C}(R)$. It means $\left(R^{n}\right)^{*} \otimes_{R} R^{n} \approx M_{n}^{C}(R)$.

Proof. Suppose that the canonical basis of $R^{n}$ is $\left\{\left(0,0, . ., 1_{i}, 0, . .0\right)\right\}_{i \in \mathbb{N}}$ and basis of $\left(R^{n}\right)^{*}$ is $\left\{\pi_{i}\right\}_{i \in \mathbb{N}}$ where $\pi_{i}\left(\left(0,0, . ., 1_{j}, 0, . .0\right)\right)=1$ for $i=j$ and 0 for $i \neq j$. Therefore

1) The comultiplication

$$
\begin{gathered}
\Delta:\left(R^{n}\right)^{*} \otimes_{R} R^{n} \rightarrow\left(\left(R^{n}\right)^{*} \otimes_{R} R^{n}\right) \otimes_{R}\left(R^{n}\right)^{*} \otimes_{R} R^{n} \\
f \otimes p \mapsto \sum_{i} f \otimes p_{i} \otimes \pi_{i} \otimes p
\end{gathered}
$$

For any $f=\sum_{i} a_{i} \pi_{i}$ and $p=\sum_{i} b_{i} p_{i} \in R^{n}$ we have

$$
\begin{aligned}
\Delta(f \otimes p) & =\sum_{k}\left(\sum_{i} a_{i} \pi_{i}\right) \otimes p_{k} \otimes \pi_{k} \otimes\left(\sum_{j} b_{j} p_{j}\right) \\
& =\sum_{k}\left(\sum_{i} a_{i} \pi_{i}\left(p_{k}\right)\right) \otimes\left(\sum_{j} b_{j} \pi_{k}\left(p_{j}\right)\right)
\end{aligned}
$$

Since $\left(R^{n}\right)^{*} \otimes_{R} R^{n} \approx M_{n}(R)$ as an $R$-module by mapping $\pi_{i} \otimes p_{j} \mapsto e_{i j}$ for any $i, j$, we have

$$
\Delta(f \otimes p) \simeq \sum_{k}\left(\sum_{i} a_{i} e_{i k} \otimes \sum_{j} b_{j} e_{k j}\right)
$$


It implies the case $f \otimes p=\pi_{i} \otimes p_{j} \approx e_{i j}$, we have

$$
\begin{aligned}
\Delta(f \otimes p) & =\Delta\left(e_{i j}\right)=\Delta\left(\pi_{i} \otimes p_{j}\right)=\sum_{k} \pi_{i} \otimes p_{k} \otimes \pi_{k} \otimes p_{j} \\
& =\sum_{k} \pi_{i}\left(p_{k}\right) \otimes \pi_{k}\left(p_{j}\right)=\sum_{k} e_{i k} \otimes e_{k j}
\end{aligned}
$$

Therefore,

$$
\Delta\left(\pi_{i} \otimes p_{j}\right) \approx \Delta\left(e_{i j}\right)=\sum_{k} e_{i k} \otimes e_{k j} .
$$

Consequently, this result similar to the comultiplication on $R$-coalgebra $M_{n}^{C}(R)$.

2) The counit of $\left(R^{n}\right)^{*} \otimes_{R} R^{n}$ is $\varepsilon(f \otimes p)=f(p)$. For any $f \otimes p \in$ $\left(R^{n}\right)^{*} \otimes_{R} R^{n}$ where $f=\sum_{i} a_{i} \pi$ and $p=\sum_{j} b_{j} p_{j} \in R^{n}$ we have

$$
\begin{aligned}
\varepsilon(f \otimes p) & =\varepsilon\left(\sum_{i} a_{i} \pi_{i} \otimes \sum_{j} b_{j} p_{j}\right)=\sum_{i} a_{i} \pi_{i}\left(\sum_{j} b_{j} p_{j}\right) \\
& =\varepsilon(f \otimes p)=\sum_{i} a_{i} \sum_{j} b_{j} \pi_{i}\left(p_{j}\right)=a_{i} b_{i} .
\end{aligned}
$$

Related with an $R$-coalgebra $M_{n}^{C}(R)$, for canonical basis $e_{i j} \approx \pi_{i} \otimes p_{j}$ (see Lemma 2). Putting $f \otimes p=\pi_{i} \otimes p_{j} \in\left(R^{n}\right)^{*} \otimes_{R} R^{n}$ (see Lemma 2), then

$$
\varepsilon\left(e_{i j}\right)=\varepsilon\left(\pi_{i} \otimes p_{j}\right)=\pi_{i}\left(p_{j}\right)=\delta_{i, j} .
$$

It is analogue to the counit of $M_{n}^{C}(R)$.

It is clear that every ring is a trivial coalgebra over itself $([15])$. On the other hand, we have already known that a ring $R$ is clean if and only if $\left(R, \Delta_{T}, \varepsilon_{T}\right)$ is a clean $R$-coalgebra. Furthermore, if $R$ is a clean ring, then the ring $M_{n}(R)$ is a clean ring [1]. Now, let consider the matrix ring $M_{n}(R)$ as a coalgebra over itself by the trivial comultiplication $\left(\Delta_{T}\right)$ and counit $\left(\varepsilon_{T}\right)$, denoted by $\left(M_{n}(R), \Delta_{T}, \varepsilon_{T}\right)$. Hence, if $M_{n}(R)$ is a clean ring, then $\left(M_{n}(R), \Delta_{T}, \varepsilon_{T}\right)$ is a clean coalgebra over itself. The following corollary explains the cleanness of $R$-coalgebra $M_{n}^{C}(R)$ with $\Delta$ and $\varepsilon$ in Equation (1) and (2).

Corollary 1. If $R$ is a clean ring, then the $R$-coalgebra $M_{n}^{C}(R)$ is clean.

Proof. By Lemma $2 M_{n}^{C}(R)$ is a special case of $P^{*} \otimes_{R} P$ when $P=R^{n}$. Suppose that $P=R^{n}$. Since $R$ is a clean ring, then $R^{n}$ is a clean $R$-module [13]. By the Theorem $3\left(R^{n}\right)^{*} \otimes_{R} R^{n}=M_{n}^{C}(R)$ is a clean $R$-coalgebra, since $R^{n}$ is a clean $R$-module. 


\section{The cleanness of $P$ and $P^{*}$ as a $P^{*} \otimes_{R} P$-comodule}

Let $R$ be a commutative ring with multiplicative identity and $P$ be a finitely generated projective $R$-module. In [15] if $P$ is a clean $R$-module, then the $R$-coalgebra $P^{*} \otimes_{R} P$ is clean. If $P$ is a finitely generated projective $R$-module with basis $p_{1}, p_{2}, \ldots, p_{n} \in P$ and dual basis $\pi_{1}, \pi_{2}, \ldots, \pi_{n} \in P^{*}$, then $P$ is a right $P^{*} \otimes_{R} P$-comodule with the coaction

$$
\varrho^{P}: P \rightarrow P \otimes_{R}\left(P^{*} \otimes_{R} P\right), p \mapsto \Sigma_{i} p_{i} \otimes \pi_{i} \otimes p .
$$

$P$ is a subgenerator in $\mathbf{M}^{P^{*} \otimes_{R} P}$ and there is a category isomorphism

$$
\mathbf{M}^{P^{*} \otimes_{R} P} \simeq \mathbf{M}_{\operatorname{End}_{R}(P)} .
$$

The dual $P^{*}$ is a left $P^{*} \otimes_{R} P$-comodule with the coaction

$$
P^{*} \varrho: P^{*} \rightarrow\left(P^{*} \otimes_{R} P\right) \otimes_{R} P^{*}, f \mapsto \Sigma_{i} f \otimes p_{i} \otimes \pi_{i} .
$$

Here, we will investigate the conditions under which $P$ and $P^{*}$ are clean comodules over $P^{*} \otimes_{R} P$.

Theorem 4. Let $P$ be a finitely generated projective $R$-module with basis $p_{1}, p_{2}, \ldots, p_{n} \in P$ and dual basis $\pi_{1}, \pi_{2}, \ldots, \pi_{n} \in P^{*}$. If $R$ is a clean ring, then $P$ is a right clean $P^{*} \otimes_{R} P$-comodule and $P^{*}$ is a left clean $P^{*} \otimes_{R} P$ comodule.

Proof. 1) Suppose that $P$ is a projective $R$-module. Consider $P$ as a right $P^{*} \otimes_{R} P$-comodule. We want to prove that $P$ is a right clean $P^{*} \otimes_{R} P$ comodule, i.e., $\left(P^{*} \otimes_{R} P\right)^{*} \operatorname{End}(P)$ is a clean ring.

Based on [15], since $P^{*} \otimes_{R} P$ is a finitely generated projective $R$ module, $R$-coalgebra $P^{*} \otimes_{R} P$ satisfies the $\alpha$-condition and we have the following condition:

$$
\left(P^{*} \otimes_{R} P\right)^{*} \mathbf{M} \simeq \mathbf{M}^{P^{*} \otimes_{R} P} .
$$

On the other hand, it is true that the ring $\left(P^{*} \otimes_{R} P\right)^{*} \simeq \operatorname{End}_{R}(P)$. Therefore,

$$
\mathbf{M}^{P^{*} \otimes_{R} P} \simeq_{\left(P^{*} \otimes_{R} P\right)^{*}} \mathbf{M} \simeq_{\operatorname{End}_{R}(P)} \mathbf{M} .
$$

We are going to prove that the ring $\left(P^{*} \otimes_{R} P\right)^{*} \operatorname{End}(P) \in_{\left(P^{*} \otimes_{R} P\right)^{*}} \mathbf{M}$ is clean. Based on Equation (3) and using the Morita Context (see Theorem 1 ), since $P$ is a generator, $R \simeq \operatorname{End}\left(\operatorname{End}_{R}(P) P\right)$ as a ring. Therefore,

$$
\left(P^{*} \otimes_{R} P\right)^{*} \operatorname{End}(P) \simeq \operatorname{End}_{\operatorname{End}_{R}(P)}(P) \text { and } \operatorname{End}_{\operatorname{End}_{R}(P)}(P) \simeq R
$$


as a ring. Hence, $\left(P^{*} \otimes_{R} P\right)^{*} \operatorname{End}(P) \simeq R$ as an $R$-module. Noted that $R$ is a clean ring if and only if $R$ is a clean $R$-module. Then

$$
\left(P^{*} \otimes_{R} P\right)^{*} \operatorname{End}(P) \simeq R
$$

is a clean ring. Consequently, $P$ is a clean $P^{*} \otimes_{R} P$-comodule.

2) Consider $P^{*}$ as a left $P^{*} \otimes_{R} P$-comodule. We want to prove that $P^{*}$ is a left $P^{*} \otimes_{R} P$-comodule, i.e., $\operatorname{End}_{\left(P^{*} \otimes_{R} P\right)^{*}}\left(P^{*}\right)$ is a clean ring. Analogue with point (1) we have

$$
P^{*} \otimes_{R} P \mathbf{M} \simeq \mathbf{M}_{\left(P^{*} \otimes_{R} P\right)^{*}} \simeq \mathbf{M}_{\operatorname{End}_{R}(P)} .
$$

We going to prove that the ring $\operatorname{End}_{\left(P^{*} \otimes_{R} P\right)^{*}}\left(P^{*}\right) \in \mathbf{M}_{\left(P^{*} \otimes_{R} P\right)^{*}}$ is clean. From Equation (3) we have

$$
\operatorname{End}_{\left(P^{*} \otimes_{R} P\right)^{*}}\left(P^{*}\right) \simeq \operatorname{End}_{\operatorname{End}_{R} P}\left(P^{*}\right) .
$$

Furthermore, from Theorem 1 we have $R \simeq \operatorname{End}_{\operatorname{End}_{R}(P)}\left(P^{*}\right)$.

Therefore,

$$
\operatorname{End}_{\left(P^{*} \otimes_{R} P\right)^{*}}\left(P^{*}\right) \simeq R .
$$

Consequently, if $R$ is a clean ring then ${ }_{\left(P^{*} \otimes_{R} P\right)^{*}} \operatorname{End}(P) \simeq R$ is a clean ring. Hence, $P^{*}$ is a left clean $P^{*} \otimes_{R} P$-comodule.

Remark 1. Let $P=R^{n}$. As a special case for any $n \in \mathbb{N}$ then $R^{n}$ is a comodule over the coalgebra $M_{n}^{C}(R)$. On the other hand, if $R$ is a clean ring (i.e., a clean $R$-module), then $R^{n}$ is a clean $R$-module. Therefore, if $R$ is a clean $R$-module, then $R^{n}$ is a right clean $M_{n}^{C}(R)$-comodule.

This paper gives the sufficient conditions of clean $R$-coalgebra $P^{*} \otimes_{R} P$ and the cleanness of $P$ and $P^{*}$ as a $P^{*} \otimes_{R} P$-comodule. We already get some conclusions i.e., if $P$ is a clean $R$-module, then the $R$-coalgebra $P^{*} \otimes_{R} P$ is clean and if $R$ is a clean ring, then $P$ (resp. $P^{*}$ ) is a right (resp. left) clean $P^{*} \otimes_{R} P$-comodule. We see that the cleanness of $P^{*} \otimes_{R} P$ depends on $R$ if $P$ is a finitely generated projective $R$-module (i.e., it is very closed to free $R$-module).

\section{References}

[1] Nicholson, W.K., Lifting Idempotents and Exchange Rings, Trans. Amer. Math. Soc., 229, 1977, 269-278.

[2] Warfield, Jr., R. B., Exchange rings and decompositions of modules, Math. Ann. 199, 1972, 31-36.

[3] Crawley, P., and Jónnson, B., Refinements for Infinite Direct Decompositions Algebraic System, Pacific J. Math., 14, 1964, 797-855. 
[4] Camillo, V.P., and Yu, H.P., Exchange Rings, Units and Idempotents, Comm. Algebra, 22(12), 1994, 4737-4749.

[5] Han, J., and Nicholson, W.K., Extension of Clean Rings, Comm. Algebra, 29(6), 2001, 2589-2595.

[6] Anderson, D.D., and Camillo, V.P., Commutative Rings Whose Element are a sum of a Unit and Idempotent, Comm. Algebra, 30(7), 2002, 3327-3336.

[7] Tousi, M., and Yassemi, S., Tensor Product of Clean Rings, Glasgow Math. J, 47. 2005, 501-503.

[8] McGovern, W. Wm., Characterization of commutative clean rings, Int. J. Math. Game Theory Algebra, 15(40), 2006, 403-413.

[9] Chen, H., and Chen, M., On Clean Ideals, IJMMS, 62, 2002, 3949-3956.

[10] Nicholson, W. K., and Varadarajan, K., Countable Linear Transformations are Clean, Proceedings of American Mathematical Socienty, 126, 1998, 61-64.

[11] Scarcoid, M.O., Perturbation the Linear Transformation By Idempotent, Irish Math. Soc. Bull., 39, 1997, 10-13.

[12] Nicholson, W.K., Varadarajan, K. and Zhou, Y., Clean Endomorphism Rings, Archiv der Mathematik, 83, 2004, 340-343.

[13] Camillo, V.P., Khurana, D., Lam, T.Y., Nicholson, W.K. and Zhou, Y., Continous Modules are Clean, J. Algebra, 304, 2006, 94-111.

[14] Camillo, V.P., Khurana, D., Lam, T.Y., Nicholson, W.K. and Zhou, Y., A Short Proof that Continous Modules are Clean, Contemporary Ring Theory 2011, Proceedings of the Sixth China-Japan-Korea International Conference on Ring Theory, 2012 165-169.

[15] Brzeziński, T., and Wisbauer, R., Corings and Comodules, Cambridge University Press, United Kingdom, 2003.

[16] Lam, T.Y., Graduated Texts in Mathematics: Lectures on Modules and Rings, Springer-Verlag, New York, inc, 1994.

[17] Adkins, W.A., and Weintraub, S. H., Algebra "An Approach via Module Theory", Springer-Verlag New York, Inc., USA, 1992.

\section{CONTACT INFORMATION}

Nikken Prima

Puspita,

Indah Emilia

Wijayanti, Budi Surodjo
Department of Mathematics, Faculty of Mathematics and Natural Science, Universitas Gadjah Mada, Yogyakarta, Indonesia E-Mail(s): nikken.prima.p@mail.ugm.ac.id (nikkenprima@gmail.com), ind_wijayanti@ugm.ac.id, surodjo_b@ugm.ac.id

Web-page(s): www.indahwijayanti.staff . ugm.ac.id, www. acadstaff.ugm.ac.id/ MTk2NTEXMjYxOTkxMDMxMDAx

Received by the editors: 10.07 .2019

and in final form 23.10.2020. 